\title{
Cuidando do cuidador: Um relato de experiência de intervenções de boas práticas de saúde para profissionais da atenção primária da linha frente na COVID-19
}

\author{
Caring for the caregiver: An experience report of good health practice interventions for frontline
} primary care professionals at COVID-19

Cuidando al cuidador: Un informe de experiencia de intervenciones de buenas prácticas de salud para profesionales de atención primaria de primera línea en COVID-19

Tereza Cristina Linhares Costa Melo

ORCID:https://orcid.org/0000-0001-8331-3552 Escola de Saúde Pública Visconde de Sabóia, Brasil E-mail: tcristinafisio11@hotmail.com

Ana Claudia Costa de Sampaio

ORCID:https://orcid.org/0000-0002-1730-8568 Escola de Saúde Pública Visconde de Sabóia, Brasil E-mail: claudiaodeon@ hotmail.com

Francisco Walter de Albuquerque Neto

ORCID:https://orcid.org/0000-0002-0854-8180 Escola de Saúde Pública Visconde de Sabóia, Brasil

E-mail: contatowalteralbuquerque@gmail.com

Jorge Luís Rodrigues dos Santos

ORCID:https://orcid.org/0000-0003-1349-6984

Escola de Saúde Pública Visconde de Sabóia, Brasil E-mail: nutrijorgeluis@gmail.com

Paloma Carvalho Alves

ORCID:https://orcid.org/0000-0002-4179-8472 Escola de Saúde Pública Visconde de Sabóia, Brasil E-mail: Paloma.ca1@hotmail.com

Lizianea de Aragão Gadelha

ORCID:https://orcid.org/0000-0003-1107-557X

Secretaria da Saúde de Sobral, Brasil

E-mail: lizi_agadelha@hotmail.com

Silvinha de Sousa Vasconcelos Costa

ORCID:https://orcid.org/0000-0002-1920-9694

Escola de Saúde Pública Visconde de Sabóia, Brasil E-mail: silvincosta@gmail.com

Carlos Romualdo de Carvalho e Araújo ORCID:https://orcid.org/0000-0001-6282-0571 Secretaria da Saúde de Sobral, Brasil E-mail: romualdocrca@hotmail.com

Ana Kelly Cândido Vasconcelos ORCID:https://orcid.org/0000-0001-7103-2219 Secretaria da Saúde de Sobral, Brasil E-mail: anakellycandido@gmail.com

Francisco Valdicélio Ferreira

ORCID: https://orcid.org/0000-0002-6347-2844

Escola de Saúde Pública Visconde de Sabóia, Brasil E-mail: celionutri@gmail.com

\begin{abstract}
Resumo
Introdução: Relata-se a experiência da intervenção em saúde denominada: "Cuidando do cuidador", junto a profissionais da equipe mínima de um Centro de Saúde da Família (CSF). A aplicação desta intervenção teve como objetivo propiciar incentivo, apoio e autocuidado aos profissionais da unidade da linha de frente da pandemia da Covid-19. Metodologia: Trata-se de estudo descritivo e qualitativo, realizado no Centro de Saúde da Família, Dr. Grijalba Mendes Carneiro, localizado em Sobral, zona Norte do Ceará, no mês de outubro de 2020. A intervenção dividiu-se em momentos de aplicação técnicas de relaxamento, com alongamentos, massagens, musicoterapia e meditação guiada, além de educação em saúde com residentes da unidade. Participaram da intervenção: agentes comunitários de saúde, enfermeiros, auxiliares administrativos, técnicos de enfermagem e médico. Resultados: Após a
\end{abstract}


intervenção, foi perceptível a diminuição da tensão destes profissionais, pois precisavam de autocuidado, relaxamento físico e mental após a sobrecarga exaustiva do trabalho na pandemia Covid-19. Os participantes relataram desejo que tais práticas acontecessem com regularidade no CSF. Conclusão: A intervenção mostrou-se relevante ao melhor bemestar físico e mental desses profissionais, ao reduzir estresse e tensão decorrentes da sobrecarga laboral pandêmica. Assim, promoveu-se autocuidado àqueles que tanto se doam para cuidar do outro.

Palavras-chave: Residência; Educação em saúde; COVID-19; Atenção primária; Boas práticas.

\begin{abstract}
Introduction: The experience of the health intervention called: "Caring for the caregiver" is reported, together with professionals from the minimum team of a Family Health Center (CSF). The application of this intervention aimed to provide encouragement, support and self-care for professionals in the front-line unit of the Covid-19 pandemic. Methodology: This is a descriptive and qualitative study, carried out at the Family Health Center, Dr. Grijalba Mendes Carneiro, located in Sobral, North of Ceará, in October 2020. The intervention was divided into moments of application relaxation techniques, with stretching, massages, music therapy and guided meditation, as well as health education with residents of the unit. Participated in the intervention: responsible health agents, nurses, administrative assistants, nursing technicians and physician. Results: After the intervention, there was a noticeable decrease in the tension of these professionals, as they needed self-care, physical and mental relaxation after the exhaustive work overload in the Covid-19 pandemic. Participants reported a desire for such practices to happen regularly at the CSF. Conclusion: The intervention proved to be relevant to the better physical and mental well-being of professionals, by reducing the stress and burden of pandemic work overload. Thus, self-care was promoted to those who give themselves so much to take care of the other.
\end{abstract}

Keywords: Residence; Health education; COVID-19; Primary attention; Good habits.

\title{
Resumen
}

Introducción: Se relata la experiencia de la intervención sanitaria denominada: "Cuidando al cuidador", junto a profesionales del equipo mínimo de un Centro de Salud de la Familia (CSF). La aplicación de esta intervención tuvo como objetivo brindar estímulo, apoyo y autocuidado a los profesionales en la unidad de primera línea de la pandemia Covid-19. Metodología: Se trata de un estudio descriptivo y cualitativo, realizado en el Centro de Salud de la Familia, Dra. Grijalba Mendes Carneiro, ubicado en Sobral, Norte de Ceará, en octubre de 2020. La intervención se dividió en momentos de aplicación de técnicas de relajación, con estiramiento, masajes, musicoterapia y meditación guiada, además de educación en salud con residentes de la unidad. Participaron de la intervención: agentes sanitarios responsables, enfermeras, auxiliares administrativos, técnicos de enfermería y médico. Resultados: Después de la intervención, hubo una notable disminución de la tensión de estos profesionales, ya que necesitaban autocuidado, relajación física y mental luego de la agotadora carga de trabajo en la pandemia Covid-19. Los participantes informaron sobre el deseo de que tales prácticas ocurrieran con regularidad en el CSF. Conclusión: La intervención demostró ser relevante para el mejor bienestar físico y mental de los profesionales, al reducir el estrés y la carga de trabajo por sobrecarga pandémica. Así, se promovió el autocuidado a quienes se entregan tanto por cuidar al otro.

Palabras clave: Residencia; Educación para la salud; COVID-19; Atención primaria; Buenas practicas.

\section{Introdução}

Os profissionais de saúde em todas as categorias enfrentam grande luta no combate à COVID-19, ficando expostos a riscos e desafios no seu dia a dia. A saúde pública tem se preparado para garantir atendimento seguro e de qualidade a pacientes e funcionários (Medeiros, 2020). As Unidades Básicas de Saúde são a principal porta de entrada do paciente no SUS e, frequentemente, o local do seu primeiro atendimento por ser a opção mais próxima e acessível ao usuário. Estas unidades são referência para o manejo dessa doença, fornecendo auxílio epidemiológico na sua vigilância e controle, e atendimento ao paciente que requer cuidado a esse processo patológico e seus determinantes (Medeiros, 2020).

Com a expansão da pandemia, as condições de trabalho em saúde se tornaram uma preocupação constante, pois há escassez de equipamentos em muitos países, hospitais e unidades básicas de saúde necessitam reorganizar seus atendimentos, qualificar profissionais e ampliar o número de consultas e internações com qualidade e segurança (Medeiros, 2020).

Assim, é inevitável que os profissionais de saúde sejam os mais vulneráveis, pois têm atuado incansavelmente na linha de frente da pandemia, lidando com seus sentimentos, estresse, receio de contrair/transmitir a doença, sensação de fracasso com o óbitos de pacientes e também de amigos e familiares, sem contar a diária incerteza sobre a doença e seu tratamento (Saidel, 2020). 
A pandemia COVID-19 tornou o cuidar mais estressante, gerando sofrimento e adoecimento psíquico. Este desgaste físico e emocional, somado à agitação da vida cotidiana do trabalhador, tem levado o profissional de saúde à exaustão psíquica e física, e trazendo como resultado inúmeras doenças, tais como: ansiedade, depressão, síndrome do pânico e síndrome de Burnout (Costa et al, 2018). Ante o exposto, faz-se necessário estimular um ambiente favorável de trabalho, em que o cuidado ao cuidador seja implantado no dia a dia das suas atividades laborais. (Moraes et al., 2016).

Em vista disso, defende-se a relevância de intervenções com técnicas de relaxamento, que reduzem dores e tensões, diminuem a ansiedade, proporcionam sentimento de controle e energia, melhoram concentração e sono, além de estimular o relacionamento com o outro, potencializando o desempenho de atividades físicas (Costa, 2018).

Com isso, percebeu-se a necessidade de proporcionar aos profissionais de saúde cuidados que focassem no processo corporal como estratégia para otimizar o equilíbrio mental, redução do estresse, ansiedade e angústia, aumento a qualidade de vida relacionada ao trabalho na linha de frente do combate à pandemia Covid-19. O objetivo neste trabalho é relatar como se deu a intervenção "Cuidando do cuidador" junto a profissionais de um CSF de Sobral com atuação na linha de frente do combate à pandemia Covid-19.

\section{Metodologia}

Este artigo compreende um relato de experiência, desenvolvido com profissionais da equipe mínima do Centro de Saúde da Família (CSF) Dr. Grijalba Mendes Carneiro, conhecido popularmente por Coelce, localizado na sede do município de Sobral-CE. A intervenção ocorreu no dia 14 de outubro de 2020, no período da manhã, das 08:00 às 11:00 horas. Inaugurado no ano de 2001, o CSF atende aos bairros: Dom José e Padre Ibiapina, com uma população adscrita de aproximadamente 10.300 habitantes, 3168 famílias (ESUS/2018).

O CSF Coelce possui quatro equipes de saúde da família, sendo cada equipe composta por: 01 médico, 01 enfermeira, 5 agentes comunitários de saúde. O CSF conta ainda com 01 gerente, 04 auxiliares administrativos, 02 vigilantes, 03 auxiliares de serviços gerais, 01 atendente de farmácia, 01 farmacêutico, 03 técnicos de enfermagem, 04 dentistas e 02 auxiliares de saúde bucal. Recebe o apoio de uma equipe multiprofissional, composta por 01 assistente social e 01 profissional de educação física, além da equipe de Residência Multiprofissional em Saúde da Família: 01 psicóloga, 01 fisioterapeuta, 01 fonoaudiólogo, 01 nutricionista e 01 docente da Escola de Saúde Pública Visconde de Saboia que atua como tutora da Residência.

O método adotado para realização do estudo consistiu na aplicação da intervenção nomeada "Cuidando do cuidador", para propiciar momentos de incentivo, apoio e autocuidado aos profissionais da unidade que atuam na linha de frente da pandemia covid 19. Foram realizadas técnicas de relaxamentos, como alongamentos, massagens, respirações profundas, musicoterapias e meditação

\section{Resultados e Discussão}

A ação foi realizada pela equipe multiprofissional de residentes em saúde da família: 01 psicóloga, 01 nutricionista, 01 fisioterapeuta, 01 fonoaudiólogo. Participaram da intervenção: 20 agentes comunitários de saúde, 3 enfermeiros, 3 auxiliares administrativos, 2 técnicos de enfermagem e 1 médico. Para o desenvolvimento da ação, foi elaborado um cronograma de técnicas de relaxamentos, primeiro foram utilizadas as técnicas de alongamentos, segundo as técnicas respiratórias com massagem, um momento de meditação, com o auxílio da musicoterapia e no final foi realizado um momento de educação em saúde com orientações quanto alimentação saudável e a importância de realizar também seu autocuidado.

A ação foi implementada em um único dia, no decorrer do expediente da unidade, os profissionais foram divididos em 
grupos de quatro pessoas, para a realização das técnicas, cada grupo teve a duração de 30 minutos. Foram realizadas com a colaboração, consentimento dos profissionais da unidade básica de saúde, cada grupo foi convidado para dirigir-se até a sala de reunião da UBS, os participantes ficaram sentados no chão em colchonetes, com distanciamento mínimo de 1 metro e meio.

A equipe multiprofissional de residentes do CSF realizou uma breve explicação de como iria prosseguir a ação, ressaltando as etapas que seriam realizadas no decorrer da dinâmica, cada membro da equipe teve um papel essencial na prática terapêutica, visto que trouxeram o olhar e o fazer de suas categorias para aplicar no momento da vivência com os profissionais da unidade de saúde.

\section{A Fisioterapeuta}

Conforme Maia et al., (2015) a atuação do profissional fisioterapeuta é importante tanto para a saúde da população do território atendido como para a equipe multiprofissional. Uma vez que este profissional não atua somente quando a deficiência ou incapacidade já está instalada, mas também na prevenção, podendo atenuar diversas patologias, diminuindo consequentemente tempo de tratamento e promovendo bem-estar físico e funcional, tanto da comunidade que atua, como dos profissionais que atendem este público.

No primeiro momento da ação foram repassados exercícios de alongamentos conduzido pela Fisioterapeuta da equipe, esses exercícios contribuíram para aliviar a tensão muscular, melhorar a postura dos participantes e aumentar a flexibilidade destes. Posteriormente foram demonstradas as técnicas de respiração profunda, contando com o auxílio da musicoterapia, que permitiu aos profissionais envolvidos na ação desenvolver um estado de profundo equilíbrio e bem-estar, contribuindo diretamente para a redução do estresse, ansiedade e sensações de dores no corpo. Após esse momento foram formadas duplas para realização da massagem relaxante, realizada por todos os residentes da equipe, quem estava realizando a massagem foi devidamente higienizado e com uso de máscara.

\section{A Psicóloga}

Seguindo a ação terapêutica, a Psicóloga ficou responsável em conduzir a meditação guiada, iniciou o momento usando a técnica Mindfulness (Atenção Plena), que consiste em alcançar um estado mental em que a consciência está focada no momento presente, enquanto calmamente se reconhece e aceita seus sentimentos, pensamentos e sensações corporais. Essa técnica conferiu ainda aos participantes a melhora de habilidades cognitivas, como a memória, criatividade e comunicação. Ao final da meditação, foi reproduzido a leitura do exercício "folhas no riacho" que despertou nos profissionais um estado de profundo relaxamento. Este exercício consistiu na leitura de um texto que guiou os participantes a deixarem todos os pensamentos estarem presentes naquele momento, sejam bons ou ruins e permitirem com que os mesmos possam seguir o fluxo junto com as folhas no riacho. Toda a prática foi realizada com o som de água corrente ao fundo.

Segundo Willhelm (2015) o profissional de psicologia ao se utilizar de técnicas de relaxamento e respiração através do Mindfulness, permite com que se promova a diminuição da ansiedade. Essas técnicas comportamentais de relaxamento ajudam nos sintomas corporais de tensão e rigidez, o que é de grande valia no ambiente laboral, uma vez que os profissionais da equipe em grande parte dos dias estão estressados e sobrecarregados com o trabalho.

\section{O Fonoaudiólogo}

A atuação da Fonoaudiologia tem ganhado destaque desde a implementação do SUS, sendo consolidada pela inserção cada vez mais presente na Atenção Primária à Saúde. Junto a equipe multidisciplinar, o profissional realiza um largo espectro de ações, tais como: acolhimento, diagnóstico situacional, visitas domiciliares, atendimentos individuais ou em grupo, salas de espera, ações de educação em saúde entre outras. Ressalta-se que quando inserido na equipe do NASF, deve priorizar o 
fortalecimento ao apoio social e o vínculo com a comunidade (Guckert; De Sousa; Arakawa-Belaunde, 2020).

A ação teve sequência com um breve discurso feito pelo Fonoaudiólogo da equipe, que reforçou a necessidade sobre os cuidados de saúde para nosso dia a dia, como cuidados de higiene, uso de roupas leves, a importância do descanso mental e físico, além de reforçar a importância do autocuidado. Na oportunidade também foram repassadas orientações gerais de fonoterapia, com o propósito de desenvolver as habilidades sociais, autonomia e fortalecimento das cordas vocais.

\section{O Nutricionista}

Ao final das etapas de relaxamento proporcionados pela prática terapêutica, o Nutricionista da equipe realizou um breve momento de educação em saúde com os profissionais da unidade, ressaltando a importância de construir hábitos alimentares saudáveis, a fim de evitar prováveis agravos e condições de adoecimento no decorrer da vida. Com o propósito de regular a ansiedade e o estresse, tão comum atualmente, foram repassadas orientações quanto ao consumo de alimentos ricos em triptofano, como abacate, castanha de caju, amendoim, banana, queijo, ovo, entre outros. Também foi instruído que os participantes consumam fontes de alimentos ricos em vitamina $\mathrm{C}$, zinco e antioxidantes, visando o fortalecimento da barreira imunitária destes, visto a necessidade latente de proteção celular em tempos de pandemia.

Segundo Costa e Santana (2011), é fundamental a inserção do profissional nutricionista na Estratégia de Saúde da Família, na busca à promoção da saúde em todas as fases e ciclos de vida, abordando aspectos da alimentação saudável, a questão da segurança alimentar, da cidadania e do direito humano fundamental à alimentação.

Durante o procedimento, foi possível perceber a diminuição da tensão, constatar que as profissionais precisavam de um descanso, deixar os pensamentos livres e relaxamento físico e mental. Além do mais os mesmos relataram necessitar de ações como essa, de forma mais frequente, pois disseram sentir necessidades desses tipos de práticas. Alguns afirmaram sentirem-se mais leves, entregando-se totalmente às atividades realizadas, outros compartilharam momentos difíceis e que no momento da meditação guiada, conseguiram relaxar e deixar a tensão de lado.

Segundo Costa et al, (2018), as técnicas de relaxamento reduzem dores e tensões, diminui à ansiedade, proporciona um sentimento de controle e energia, melhora a concentração e o sono, além de estimular o relacionamento com o outro, potencializa o desempenho de atividades físicas. 
Figura 1: A Importância da Equipe Multiprofissional.

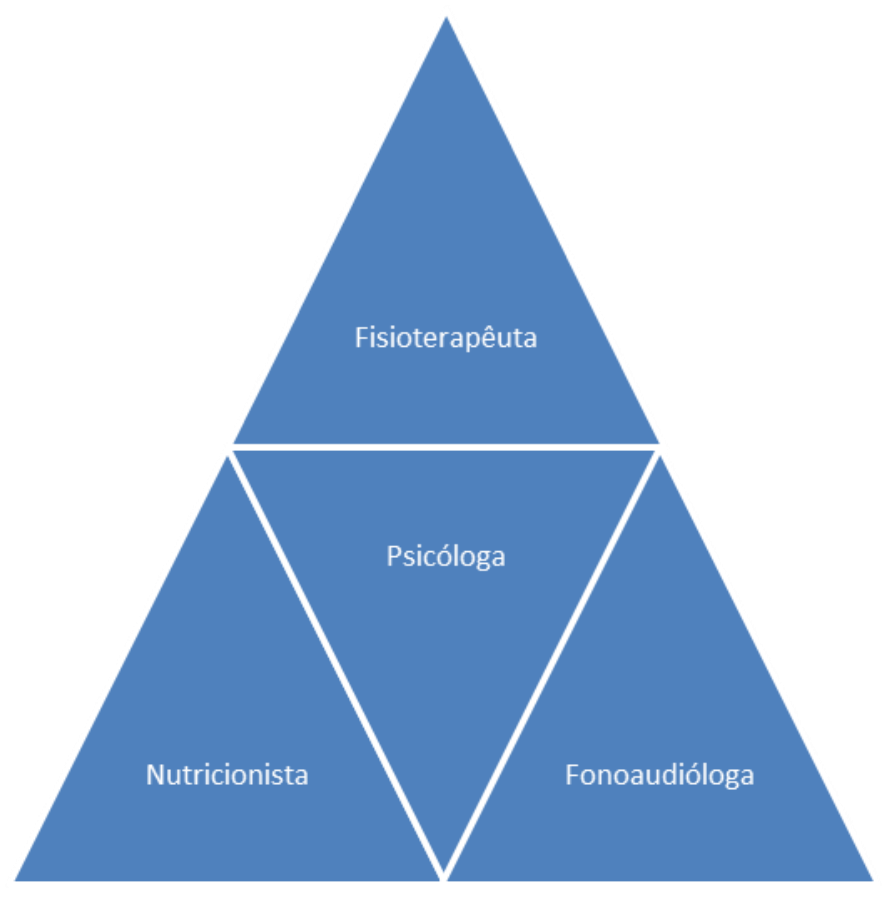

Fonte: Elaborado pelos Autores.

O fortalecimento da equipe multiprofissional e suas ações integradas contribui de forma significativa no fazer saúde, visto que o mais beneficiado é o usuário do Sistema Único de Saúde, com isto Saar e Trevizan (2007), descartam que a multiprofissionalidade é uma estratégia de fortalecimento que orienta e possibilita a realização de uma assistência integral com foco nas diversas necessidades no cotidiano do trabalho e no território.

A equipe multiprofissional neste contexto confirma mais ainda a sua importância no fazer saúde de forma holística, destacando aqui o fazer dos profissionais Fisioterapeutas, Psicólogos, Nutricionistas e Fonoaudiólogos como componentes desta ação, corroborando ainda com essa importância entre os profissionais da saúde, Nascimento e Omena (2021) destacam que a multiprofissionalidade é necessário, assim como a qualificação no serviço para trabalhar nas questões da integralidade, humanização abrangendo as varias necessidades encontradas nas áreas de atuação.

\section{Conclusão}

A criação de um ambiente de cuidado ao trabalhador foi importante, pois pode proporcionar a esses profissionais de saúde momentos mais humanizados e acolhedores diante do cenário atípico e conturbado vivenciado.

Com a realização dessa intervenção, pode-se observar que os profissionais de saúde conseguiram tirar alguns momentos de uma rotina cansativa de trabalho para ter um olhar particularizado para si mesmo, proporcionando autocuidado.

Buscou-se, assim, contribuir para qualidade de vida desses profissionais, seu bem-estar físico e mental, além de favorecer o atendimento da população por um profissional com menos estresse e tensão. Dessa forma, foi possível promover cuidado àqueles que tanto se doam para cuidar do outro.

\section{Referências}

Costa, L. K., Nunes, C. S. B. L., Lima, A. T., Barreto, D. I. T., Gonçalves, N. C. C. F., \& Aguiar, S. P. Y. (2018). Relaxadamente: "respira, inspira e não pira"implantação das técnicas de relaxamento na Atenção Básica. Revista Interdisciplinar - Centro universitário Uninovafapi, 11(3), $136-144$. 
Research, Society and Development, v. 10, n. 5, e14110515007, 2021

(CC BY 4.0) | ISSN 2525-3409 | DOI: http://dx.doi.org/10.33448/rsd-v10i5.15007

Costa, K. A. O., \& Santana, P. R. (2011). A Importância e o papel do nutricionista na Atenção Básica em Vitória de Santo Antão/PE. Revista Eletrônica Tempus, 5(4), 66-85.

Guckert, S. B., Souza, C. R. de., \& Arakawa-Belaunde, A. M. (2020). Atuação fonoaudiológica na atenção básica na perspectiva de profissionais dos núcleos de apoio à saúde da família. CoDAS, 32(5), 1-8.

Lopes, D. M. Q., Lunardi Filho, W. D., Beck, C. L. C., \& Coelho, C. (2018). Cargas de trabalho do Agente Comunitário de Saúde: Pesquisa e Assistência na Perspectiva Covergente - Assistencial. Texto contexto - enferm, 27(4), 2-10.

Maia, F., Moura, E., Madeiros, E., Carvalho, R., Silva, S., \& Santos, G. (2015). A importância da inclusão do profissional fisioterapeuta na atenção básica de Saúde. Revista da Faculdade de Ciências Médicas de Sorocaba, 17(3), 110-115.

Medeiros, E. A. S. (2020). Os profissionais de saúde enfrentam a COVID-19 nos hospitais e expõe a própria vida para cuidar dos doentes. https://pressreleases.scielo.org/blog/2020/07/03/os-profissionais-de-saude-enfrentam-a-covid-19-nos-hospitais-e-expoe-a-propria-vida-para-cuidar-dosdoentes.

Moraes, F., Benetti, E. R. R., Herr, G. E. G., Stube, M., Stumm, E. M. F., \& Guido, L. A. (2016). Estratégias de coping utilizadas por trabalhadores de Enfermagem em terapia intensiva neonatal. REME-Rev Min Enferm, 20:e966, 1-7.

Nascimento, A. C. B. do., \& Omena, K. V. M. de. (2021). Interprofessional Education in Multiprofessional Residency Programs in Health in Brazil: An integrative review. Research, Society and Development, 10(4), e8010413655.

Saidel, M. G. B., Lima, M. H. M., Campos, C. J. G., Loyola, C. M. D., Esperidião, E., \& Santos, J. R. (2020). Intervenções em saúde mental para profissionais de saúde frente a pandemia de Coronavírus. Revista enfermagem UERJ, 28, e49923, 1-6.

Saar, S. R. da C., \& Trevizan, M. A. (2007). Papéis profissionais de uma equipe de saúde: visão de seus integrantes. Revista Latino-Americana de Enfermagem, 15(1), 106-112.

Willhelm, A. R., Andretta, I., \& Ungaretti, M. S. (2015). Importância das técnicas de relaxamento na terapia cognitiva para ansiedade. Contextos Clínic, São Leopoldo, 8(1), 79-86. 